\title{
Spin-Dependent Coupled Altarelli-Parisi Equations in the NLO and the Method of Successive Approximations
}

\author{
Ranjit Choudhury' ${ }^{1}$ and D. K. Choudhury ${ }^{2}$ \\ ${ }^{1}$ Department of Physics, Goalpara College, Assam, Goalpara 783101, India \\ ${ }^{2}$ Department of Physics, Gauhati University, Assam, Guwahati 781014, India \\ Correspondence should be addressed to Ranjit Choudhury, rabrchran@yahoo.co.in
}

Received 28 April 2012; Accepted 28 May 2012

Academic Editors: F. R. Joaquim, S. Oh, and S. Paganis

Copyright (c) 2012 R. Choudhury and D. K. Choudhury. This is an open access article distributed under the Creative Commons Attribution License, which permits unrestricted use, distribution, and reproduction in any medium, provided the original work is properly cited.

The coupled Altarelli-Parisi (AP) equations for polarized singlet quark distribution and polarized gluon distribution, when considered in the small $x$ limit of the next to leading order (NLO) splitting functions, reduce to a system of two first order linear nonhomogeneous integrodifferential equations. We have applied the method of successive approximations to obtain the solutions of these equations. We have applied the same method to obtain the approximate analytic expressions for spin-dependent quark distribution functions with individual flavour and polarized structure functions for nucleon.

\section{Introduction}

The study of the evolution of the quark and gluon contributions at small $x$ (Bjorken variable) towards the spin of the proton through Altarelli-Parisi equations (AP) [1-3] is an important area of DIS. There are no data below $x \approx 0.005$ and as a result polarized gluon distribution $\Delta G(x)$ is basically unconstrained at small $x$. There are theoretical arguments that polarized gluon distribution $\Delta G(x)$ and the unpolarized gluon distribution $G(x)$ are connected through the relation $\Delta G(x) \approx x G(x)$ at small $x$, but they cannot be verified due to lack of data. Precise measurement of the polarized structure function $g_{1}\left(x, Q^{2}\right)$ and its logarithmic scale dependence can determine $\Delta G(x)$ at small $x$ and thus it can reduce the extrapolation uncertainties of $\Delta G(x)$ in the integral $\int_{0}^{1} \Delta G\left(x, Q^{2}\right) d x$ entering in the proton spin sum rule. EIC $[4,5]$ will allow for a determination of $\Delta G(x)$ down to a very small value of $10^{-4}$ and it will eventually give the gluon contribution to the spin of the proton over all $x$ to about 10 percent accuracy. The same set of measurements will also provide a significantly better determination of the total quark contribution $\Delta \Sigma[5]$. 
The Jacobi polynomial method [5-9] is one of the important methods for obtaining the solutions of spin-dependent Altarelli-Parisi equations. The main advantage of this method is that it allows us to factorize the $x$ and $Q^{2}$ dependence of the structure function in a manner that allows an efficient parameterization and evolution of the structure function. The method of successive approximations is used to solve the integral equation [10]. In such a method one begins with a crude approximation to a solution by using an initial condition and improves it step by step by applying a repeatable operation (Picard's method of successive approximations). In this work, we have applied this method for obtaining the solutions of the spin-dependent integrodifferential coupled Altarelli-Parisi equations at small $x$ in the $\mathrm{NLO}$ and we begin the process by using the boundary condition that the parton distribution vanishes at $x=1$ [11-13]. We have shown that the application of this method in solving these equations results in solutions which appear as summation of series, each term of which is the product of $x$ and $Q^{2}$-dependent functions.

We have structured our work as follows: in Section 2, we have given a method of solution of a system of two first order linear homogeneous differential equations with variable coefficients under certain conditions. In Section 3, we have shown that in the small $x$ limit of the splitting functions and under some reasonable approximation of the coupling constant, the AP equations for polarized parton distributions become two first order simultaneous linear nonhomogeneous integrodifferential equations. By using the method described in Section 2 and the method of variation [10], we have shown that the solutions can be improved through successive approximations. The same procedure is applied to obtain the approximate analytical expressions for polarized quark distributions with individual flavour and using them we have obtained the expressions for polarized structure functions for proton $g_{1}^{p}\left(x, Q^{2}\right)$ and as well as for neutron $g_{1}^{n}\left(x, Q^{2}\right)$. We have compared our solutions with some numerically obtained solutions.

\section{Method of Solving a System of FLHE}

A system of two first order linear homogenous differential equations (FLHE) with variable coefficients can be given as

$$
\begin{aligned}
& \frac{d f(t)}{d t}=a_{1}(t) \times f(t)+b_{1}(t) \times g(t), \\
& \frac{d g(t)}{d t}=a_{2}(t) \times f(t)+b_{2}(t) \times g(t),
\end{aligned}
$$

where $a_{1}(t), b_{1}(t), a_{2}(t)$, and $b_{2}(t)$ are known coefficients, $f(t)$ and $g(t)$ are unknown functions to be determined, and $t$ is the independent variable.

Equations (2.1) and (2.2) are analytically solvable if the coefficients $a_{i}$ and $b_{i}, i=1,2$, are constants, that is, independent of $t$ [10]. But, as noted in [10], there is no general method of solving such a system of equations when the coefficients are not constant.

Here we shall present a method of solving (2.1) and (2.2) when $a_{i}(t)=a_{i} T(t)$ and $b_{i}(t)=b_{i} T(t), i=1,2$ have got identical $t$ dependence $T(t)$. 
Our system of (2.1) and (2.2) can be written as

$$
\begin{aligned}
& \frac{d f}{f}=T(t)\left(a_{1}+b_{1} \frac{g}{f}\right) d t \\
& \frac{d g}{g}=T(t)\left(a_{2} \frac{f}{g}+b_{2}\right) d t .
\end{aligned}
$$

Integrating (2.3), we have

$$
\begin{aligned}
& \ln f=\int T(t)\left(a_{1}+b_{1} \frac{g}{f}\right) d t-\ln c_{1}, \\
& \ln g=\int T(t)\left(a_{2} \frac{f}{g}+b_{2}\right) d t-\ln c_{2},
\end{aligned}
$$

where $c_{1}$ and $c_{2}$ are constants of integration.

Substracting (2.5) from (2.4), we have

$$
\ln \frac{f}{g}=\int T(t)\left(a_{1}+b_{1} \frac{g}{f}-a_{2} \frac{f}{g}-b_{2}\right) d t+\ln \frac{c_{2}}{c_{1}}
$$

or

$$
\ln \frac{u}{u_{0}}=\int T(t)\left(a_{1}+\frac{b_{1}}{u}-a_{2} u-b_{2}\right) d t,
$$

where

$$
\begin{aligned}
& u_{0}=\frac{c_{2}}{c_{1}}, \\
& u=\frac{f}{g} .
\end{aligned}
$$

Differentiating (2.7) with respect to $t$, we have

$$
\frac{d u}{d t}=T(t)\left(a_{1} u+b_{1}-a_{2} u^{2}-b_{2} u\right)
$$

leading to

$$
\frac{1}{a_{2}\left(\lambda_{q}-\lambda_{p}\right)}\left[\frac{d u}{u-\lambda_{p}}-\frac{d u}{u-\lambda_{q}}\right]=T(t) d t
$$

where

$$
\lambda_{p, q}=\frac{\left(b_{2}-a_{1}\right) \pm \sqrt{\left(a_{1}-b_{2}\right)^{2}+4 a_{2} b_{1}}}{-2 a_{2}} .
$$


Integrating (2.10), we have

$$
\ln \left(u-\lambda_{p}\right)-\ln \left(u-\lambda_{q}\right)=a_{2}\left(\lambda_{q}-\lambda_{p}\right) \int T(t) d t+\ln c,
$$

where $\ln c$ is the integration constant.

From (2.12) we have

$$
u=\frac{f}{g}=\frac{\lambda_{p}-\lambda_{q} c \exp \left[n \int T(t) d t\right]}{1-c \exp \left[n \int T(t) d t\right]},
$$

where $n=a_{2}\left(\lambda_{q}-\lambda_{p}\right)$.

Equation (2.13) implies that we can write

$$
\begin{gathered}
f(t)=K(t)\left[\lambda_{p}-\lambda_{q} c \exp \left(n \int T(t) d t\right)\right], \\
g(t)=K(t)\left[1-c \exp \left(n \int T(t) d t\right)\right],
\end{gathered}
$$

where $K(t)$ is a function of $t$ to be determined.

We now put (2.14) in (2.1) and obtain

$$
\frac{d K(t)}{K}=\frac{\left(a \exp \left(n \int T(t) d t\right)+b\right)}{\lambda_{p}-\lambda_{q} c \exp \left(n \int T(t) d t\right)} T(t) d t,
$$

where

$$
\begin{gathered}
a=c\left(n \lambda_{q}-a_{1} \lambda_{q}-b_{1}\right), \\
b=a_{1} \lambda_{p}+b_{1} .
\end{gathered}
$$

Integrating (2.15), we have

$$
K(t)=H_{0} \exp \left[\left(a_{1}+\frac{b_{1}}{\lambda_{p}}\right) \int T(t) d t\right]
$$

where $H_{0}$ is the constant of integration. From (2.14) we can now obtain the expression for $f(t)$ and $g(t)$. 


\section{Spin-Dependent AP Equations and Polarized Structure Functions in NLO}

\subsection{Altarelli-Parisi Equations}

The coupled Altarelli-Parisi equations [1-3] for polarized singlet quark density, polarized gluon density, and polarized individual quark density are given as

$$
\begin{aligned}
\frac{\partial \Delta \Sigma(x, t)}{\partial t}= & \frac{\alpha_{s}(t)}{2 \pi} \int_{x}^{1} \frac{d z}{z} \Delta P_{q q}\left(\frac{x}{z}\right) \Delta \Sigma(z, t) \\
& +\frac{\alpha_{s}(t)}{2 \pi} \int_{x}^{1} \frac{d z}{z} \Delta P_{q g}\left(\frac{x}{z}\right) \Delta G(z, t), \\
\frac{\partial \Delta G(x, t)}{\partial t}= & \frac{\alpha_{s}(t)}{2 \pi} \int_{x}^{1} \frac{d z}{z} \Delta P_{g q}\left(\frac{x}{z}\right) \Delta \Sigma(z, t) \\
& +\frac{\alpha_{s}(t)}{2 \pi} \int_{x}^{1} \frac{d z}{z} \Delta P_{g g}\left(\frac{x}{z}\right) \Delta G(z, t), \\
\frac{\partial \Delta q_{i}(x, t)}{\partial t}= & \frac{\alpha_{s}(t)}{2 \pi} \int_{x}^{1} \frac{d z}{z} \Delta P_{q q}\left(\frac{x}{z}\right) \Delta q_{i}(z, t) \\
& +\frac{\alpha_{s}(t)}{2 \pi} \int_{x}^{1} \frac{d z}{z} \frac{\Delta P_{q g}(x / z)}{2 n_{f}} \Delta G(z, t) .
\end{aligned}
$$

The polarized splitting functions $\Delta P_{i j}(x)$ are defined as

$$
\Delta P_{i j}(x)=\Delta P_{i j}^{(0)}(x)+\frac{\alpha_{S}(t)}{2 \pi} \Delta P_{i j}^{(1)}(x) .
$$

$\Delta P_{i j}^{(0)}(x)$ and $\Delta P_{i j}^{(1)}(x)$ are given in $[14,15]$.

$\Delta P_{i j}^{(0)}(x)$ in the small $x$ limit are given as [16]

$$
\begin{gathered}
\Delta P_{q q}^{(0)} x=\frac{4}{3}\left[1+\frac{1}{2} \delta(1-x)\right], \\
\Delta P_{q g}^{(0)} x=n_{f}[-1+2 \delta(1-x)], \\
\Delta P_{g q}^{(0)} x=\frac{4}{3}[2-\delta(1-x)], \\
\Delta P_{g g}^{(0)} x=3\left[4-\frac{13}{6} \delta(1-x)\right]-\frac{n_{f}}{3} \delta(1-x)
\end{gathered}
$$


and $\Delta P_{i j}^{(1)}(x)$ in the small $x$ limit can be given as $[14,15]$

$$
\begin{aligned}
& \Delta P_{q q}^{(1)}(x)=\Delta P_{q q 0}\left(n_{f}\right)+\Delta P_{q q 1}\left(n_{f}\right) \ln x+\Delta P_{q q 2}\left(n_{f}\right) \ln ^{2} x, \\
& \Delta P_{q g}^{(1)}(x)=\Delta P_{q g 0}\left(n_{f}\right)+\Delta P_{q g 1}\left(n_{f}\right) \ln x+\Delta P_{q g 2}\left(n_{f}\right) \ln ^{2} x, \\
& \Delta P_{g q}^{(1)}(x)=\Delta P_{g q 0}\left(n_{f}\right)+\Delta P_{g q 1}\left(n_{f}\right) \ln x+\Delta P_{g q 2}\left(n_{f}\right) \ln ^{2} x, \\
& \Delta P_{g g}^{(1)}(x)=\Delta P_{g g 0}\left(n_{f}\right)+\Delta P_{g g 1}\left(n_{f}\right) \ln x+\Delta P_{g g 2}\left(n_{f}\right) \ln ^{2} x,
\end{aligned}
$$

where $\Delta P_{a b i}, a=q, g ; b=q, g$ and $i=0,1,2$ are given in the appendix.

$\alpha_{s}(t)$, the running coupling constant of QCD in NLO, is defined as

$$
\alpha_{S}(t)=\frac{4 \pi}{\beta_{0} t}\left(1-\frac{\beta_{1} \ln t}{\beta_{0}^{2} t}\right)
$$

where

$$
\begin{aligned}
& \beta_{0}=11-\frac{2 n_{f}}{3} \\
& \beta_{1}=102-\frac{38 n_{f}}{3}
\end{aligned}
$$

and $n_{f}$ is the number of active flavours.

We define

$$
T(t)=\frac{\alpha_{s}(t)}{2 \pi} .
$$

To proceed further and to apply our formalism, we, as in [17], use the assumption

$$
T(t)^{2}=T_{0} T(t)
$$

where $T_{0}$ is a numerical parameter. 


\subsection{Solutions of AP Equations for $\Delta \Sigma(x, t)$ and $\Delta G(x, t)$ in NLO}

We first solve (3.1) and (3.2) for obtaining the approximate analytic expressions for $\Delta \Sigma(x, t)$ and $\Delta G(x, t)$ in NLO. Using the assumption (3.10) and the small $x$ splitting functions in NLO, these equations can be written as

$$
\begin{aligned}
\frac{\partial \Delta \Sigma(x, t)}{\partial t}=T(t)( & a_{1} \Delta \Sigma(x, t)+b_{1} \Delta G(x, t) \\
& +h_{1}(x) \int_{x}^{1} \frac{d z}{z} \Delta \Sigma(z, t)+h_{2}(x) \int_{x}^{1} \frac{d z}{z} \ln z \Delta \Sigma(z, t) \\
& +h_{3}(x) \int_{x}^{1} \frac{d z}{z} \ln ^{2} z \Delta \Sigma(z, t)+k_{1}(x) \int_{x}^{1} \frac{d z}{z} \Delta G(z, t) \\
& \left.+k_{2}(x) \int_{x}^{1} \frac{d z}{z} \ln z \Delta G(z, t)+k_{3}(x) \int_{x}^{1} \frac{d z}{z} \ln ^{2} z \Delta G(z, t)\right), \\
\frac{\partial \Delta G(x, t)}{\partial t}=T(t)( & a_{2} \Delta \Sigma(x, t)+b_{2} \Delta G(x, t) \\
& +p_{1}(x) \int_{x}^{1} \frac{d z}{z} \Delta \Sigma(z, t)+p_{2}(x) \int_{x}^{1} \frac{d z}{z} \ln z \Delta \Sigma(z, t) \\
& +p_{3}(x) \int_{x}^{1} \frac{d z}{z} \ln ^{2} z \Delta \Sigma(z, t)+q_{1}(x) \int_{x}^{1} \frac{d z}{z} \Delta G(z, t) \\
& \left.+q_{2}(x) \int_{x}^{1} \frac{d z}{z} \ln z \Delta G(z, t)+q_{3}(x) \int_{x}^{1} \frac{d z}{z} \ln ^{2} z \Delta G(z, t)\right) .
\end{aligned}
$$

$a_{1}, b_{1}, a_{2}, b_{2}$ are some known constants and $h_{i}(x), k_{i}(x), p_{i}(x)$ and $q_{i}(x), i=1,2,3$ are known functions of $x$.

As described in [10], we obtain the first approximate solutions of (3.11) by replacing $\Delta \Sigma(x, t)$ and $\Delta G(x, t)$ under the integrals appearing in the right-hand side of these equations by their boundary values at $x=1$ [11-13]:

$$
\begin{aligned}
& \left.\Delta \Sigma(x, t)\right|_{x=1}=0, \\
& \left.\Delta G(x, t)\right|_{x=1}=0 .
\end{aligned}
$$

With these substitutions (3.11) become

$$
\begin{aligned}
& \frac{\partial \Delta \Sigma(x, t)}{\partial t}=a_{1} T(t) \Delta \Sigma(x, t)+b_{1} T(t) \Delta G(x, t), \\
& \frac{\partial \Delta G(x, t)}{\partial t}=a_{2} T(t) \Delta \Sigma(x, t)+b_{2} T(t) \Delta G(x, t) .
\end{aligned}
$$


Equations (3.13) are two first order simultaneous linear homogeneous differential equations with variable coefficients. We solve these equations by the method described in Section 2 and find the solutions as

$$
\begin{gathered}
\Delta \Sigma(x, t)=\lambda_{2} \Theta_{1} e^{N_{1}\left(n_{f}\right) \tau(t)}-\lambda_{1} \Theta_{2} e^{N_{2}\left(n_{f}\right) \tau(t)}, \\
\Delta G(x, t)=\Theta_{1} e^{N_{1}\left(n_{f}\right) \tau(t)}-\Theta_{2} e^{N_{2}\left(n_{f}\right) \tau(t)},
\end{gathered}
$$

where

$$
\begin{gathered}
\lambda_{1}=\frac{\left(b_{2}-a_{1}\right)+\sqrt{\left(a_{1}-b_{2}\right)^{2}+4 b_{1} a_{2}}}{-2 a_{2}}, \\
\lambda_{2}=\frac{\left(b_{2}-a_{1}\right)-\sqrt{\left(a_{1}-b_{2}\right)^{2}+4 b_{1} a_{2}}}{-2 a_{2}}, \\
N_{1}\left(n_{f}\right)=a_{1}+\frac{b_{1}}{\lambda_{1}}+a_{2}\left(\lambda_{2}-\lambda_{1}\right), \\
N_{2}\left(n_{f}\right)=a_{1}+\frac{b_{1}}{\lambda_{1}} \\
\tau(t)=\int T(t) d t
\end{gathered}
$$

and $\Theta_{1}$ and $\Theta_{2}$ are constants of integration. From now on we shall represent $N_{i}\left(n_{f}\right)$ by $N_{i}$ and $\tau(t)$ by $\tau$.

Now applying the input distributions at $t=t_{0}$,

$$
\begin{aligned}
& \left.\Delta \Sigma(x, t)\right|_{t=t_{0}}=\Delta \Sigma\left(x, t_{0}\right), \\
& \left.\Delta G(x, t)\right|_{t=t_{0}}=\Delta G\left(x, t_{0}\right),
\end{aligned}
$$

we can find out the constants of integration $\Theta_{1}$ and $\Theta_{2}$. With these the solutions after first approximation become

$$
\begin{aligned}
& \Delta \Sigma_{1}(x, t)=U_{10}(x) \exp \left[N_{1}\left(\tau-\tau_{0}\right)\right]-\tilde{U}_{10}(x) \exp \left[N_{2}\left(\tau-\tau_{0}\right)\right] \\
& \Delta G_{1}(x, t)=V_{10}(x) \exp \left[N_{1}\left(\tau-\tau_{0}\right)\right]-\tilde{V}_{10}(x) \exp \left[N_{2}\left(\tau-\tau_{0}\right)\right]
\end{aligned}
$$


where

$$
\begin{gathered}
U_{10}(x)=\lambda_{2}\left(\frac{\Delta \Sigma\left(x, t_{0}\right)-\lambda_{1} \Delta G\left(x, t_{0}\right)}{\left(\lambda_{2}-\lambda_{1}\right)}\right) \\
\tilde{U}_{10}(x)=\lambda_{1}\left(\frac{\Delta \Sigma\left(x, t_{0}\right)-\lambda_{2} \Delta G\left(x, t_{0}\right)}{\left(\lambda_{2}-\lambda_{1}\right)}\right) \\
V_{10}(x)=\left(\frac{\Delta \Sigma\left(x, t_{0}\right)-\lambda_{1} \Delta G\left(x, t_{0}\right)}{\left(\lambda_{2}-\lambda_{1}\right)}\right) \\
\tilde{V}_{10}(x)=\left(\frac{\Delta \Sigma\left(x, t_{0}\right)-\lambda_{2} \Delta G\left(x, t_{0}\right)}{\left(\lambda_{2}-\lambda_{1}\right)}\right) \\
\tau_{0}=\left.\left(\int T d t\right)\right|_{t=t_{0}}{ }^{\prime}
\end{gathered}
$$

and subscript 1 of $\Delta \Sigma_{1}(x, t)$ and $\Delta G_{1}(x, t)$ in (3.17) refers to the first approximate solutions.

Now using the expressions (3.17) for $\Delta \Sigma_{1}(x, t)$ and $\Delta G_{1}(x, t)$ in the places of $\Delta \Sigma(x, t)$ and $\Delta G(x, t)$ appearing under the integrals in the right-hand side of (3.11), we have

$$
\begin{aligned}
\frac{\partial \Delta \Sigma(x, t)}{\partial t}= & a_{1} T(t) \Delta \Sigma(x, t)+b_{1} T(t) \Delta G(x, t) \\
& +H_{10}(x) T(t) e^{N_{1}\left(\tau-\tau_{0}\right)}-\widetilde{H}_{10}(x) T(t) e^{N_{2}\left(\tau-\tau_{0}\right)}, \\
\frac{\partial \Delta G(x, t)}{\partial t}= & a_{2} T(t) \Delta \Sigma(x, t)+b_{2} T(t) \Delta G(x, t) \\
& +K_{10}(x) T(t) e^{N_{1}\left(\tau-\tau_{0}\right)}-\widetilde{K}_{10}(x) T(t) e^{N_{2}\left(\tau-\tau_{0}\right)}
\end{aligned}
$$

where $H_{10}(x), \widetilde{H}_{10}, K_{10}(x)$, and $\widetilde{K}_{10}(x)$ are known functions of $x$.

The solutions of the homogeneous parts of (3.19), that is, the solutions of the first order linear coupled homogeneous equation (3.13) can be obtained by the method described earlier and the solutions are given as (3.14). Now, to obtain the solutions of the nonhomogeneous coupled equation (3.19) we apply the method of variation [10]. Thus the solutions of (3.19) can be given as

$$
\begin{aligned}
\Delta \Sigma_{2}(x, t)= & {\left[U_{20}(x)+U_{21}(x)\left(\tau-\tau_{0}\right)\right] \exp \left[N_{1}\left(\tau-\tau_{0}\right)\right] } \\
& -\left[\tilde{U}_{20}(x)+\tilde{U}_{21}(x)\left(\tau-\tau_{0}\right)\right] \exp \left[N_{2}\left(\tau-\tau_{0}\right)\right], \\
\Delta G_{2}(x, t)= & {\left[V_{20}(x)+V_{21}(x)\left(\tau-\tau_{0}\right)\right] \exp \left[N_{1}\left(\tau-\tau_{0}\right)\right] } \\
& -\left[\tilde{V}_{20}(x)+\tilde{V}_{21}(x)\left(\tau-\tau_{0}\right)\right] \exp \left[N_{2}\left(\tau-\tau_{0}\right)\right] .
\end{aligned}
$$

$U_{20}(x), U_{21}(x), V_{20}(x), V_{21}(x)$ and their tilde counterparts are known functions of $x$. Equations (3.20) and (3.21) are the second iterative solutions of (3.11) and in comparison to the first iterative solutions (3.17), they are closer to the numerical results as seen from Figures 1 and 2. 


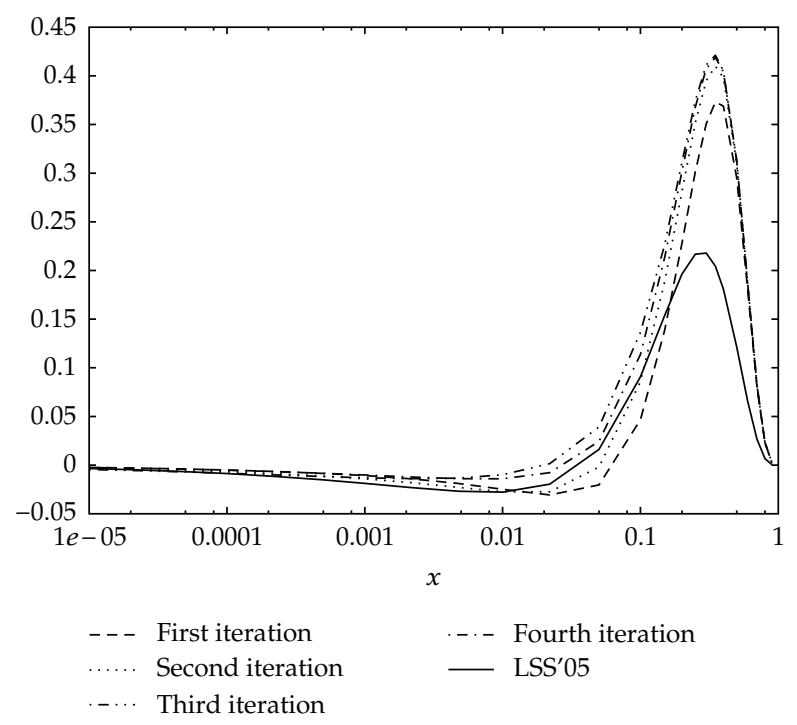

Figure 1: $x \Delta \sum\left(x, Q^{2}\right)$ at $10 \mathrm{GeV}^{2}$ at NLO.

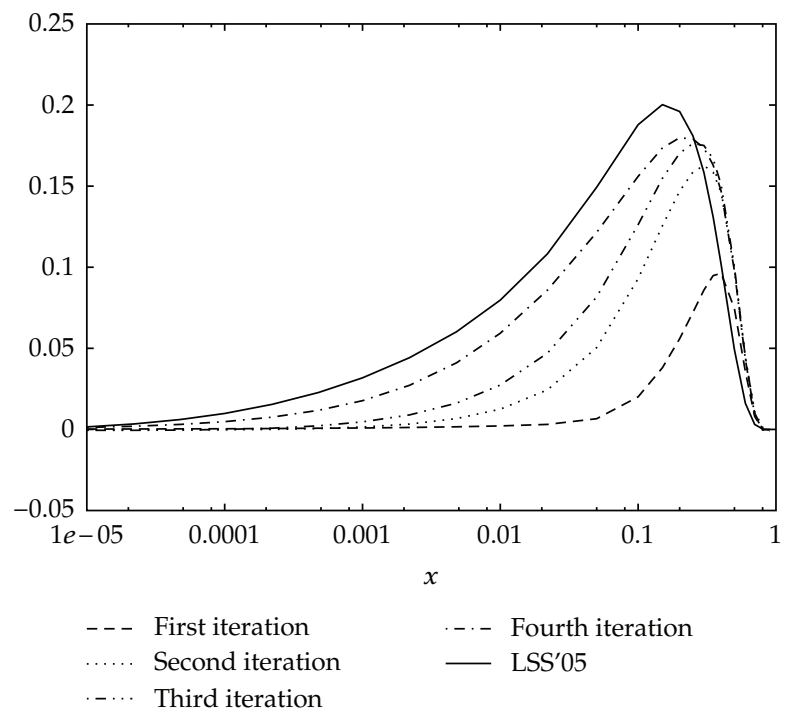

Figure 2: $x \Delta G\left(x, Q^{2}\right)$ at $10 \mathrm{GeV}^{2}$ at NLO.

We again substitute $\Delta \Sigma_{2}(x, t)$ and $\Delta G_{2}(x, t)$ from (3.20), and (3.21) respectively, for the $\Delta \Sigma(x, t)$ and $\Delta G(x, t)$ appearing in the integrals in the right-hand side of (3.11) and the resulting equations can be written as

$$
\begin{aligned}
\frac{\partial \Delta \Sigma(x, t)}{\partial t}= & a_{1} T(t) \Delta \Sigma(x, t)+b_{1} T(t) \Delta G(x, t) \\
& +\left[H_{20}(x)+H_{21}(x)\left(\tau-\tau_{0}\right)\right] T(t) e^{N_{1}\left(\tau-\tau_{0}\right)} \\
& -\left[\widetilde{H}_{20}(x)+\widetilde{H}_{21}(x)\left(\tau-\tau_{0}\right)\right] T(t) e^{N_{2}\left(\tau-\tau_{0}\right)},
\end{aligned}
$$


ISRN High Energy Physics

$$
\begin{aligned}
\frac{\partial \Delta G(x, t)}{\partial t}= & a_{2} T(t) \Delta \Sigma(x, t)+b_{2} T(t) \Delta G(x, t) \\
& +\left[K_{20}(x)+K_{21}(x)\left(\tau-\tau_{0}\right)\right] T(t) e^{N_{1}\left(\tau-\tau_{0}\right)} \\
& -\left[\widetilde{K}_{20}(x)+\widetilde{K}_{21}(x)\left(\tau-\tau_{0}\right)\right] T(t) e^{N_{2}\left(\tau-\tau_{0}\right)} .
\end{aligned}
$$

$H_{20}(x), H_{21}(x), K_{20}(x)$ and $K_{21}(x)$ and their tilde versions as appearing in (3.22) are known functions of $x$.

Proceeding in a similar manner we can obtain the solutions of (3.22) as

$$
\begin{aligned}
\Delta \Sigma_{3}(x, t)= & {\left[U_{30}(x)+U_{31}(x)\left(\tau-\tau_{0}\right)+U_{32}(x) \frac{\left(\tau-\tau_{0}\right)^{2}}{2}\right] \exp \left[N_{1}\left(\tau-\tau_{0}\right)\right] } \\
& -\left[\tilde{U}_{30}(x)+\tilde{U}_{31}(x)\left(\tau-\tau_{0}\right)+\tilde{U}_{32}(x) \frac{\left(\tau-\tau_{0}\right)^{2}}{2}\right] \exp \left[N_{2}\left(\tau-\tau_{0}\right)\right], \\
\Delta G_{3}(x, t)= & {\left[V_{30}(x)+V_{31}(x)\left(\tau-\tau_{0}\right)+V_{32}(x) \frac{\left(\tau-\tau_{0}\right)^{2}}{2}\right] \exp \left[N_{1}\left(\tau-\tau_{0}\right)\right] } \\
& -\left[\tilde{V}_{30}(x)+\tilde{V}_{31}(x)\left(\tau-\tau_{0}\right)+\tilde{V}_{32}(x) \frac{\left(\tau-\tau_{0}\right)^{2}}{2}\right] \exp \left[N_{2}\left(\tau-\tau_{0}\right)\right] .
\end{aligned}
$$

The expressions for $U_{3 i}(x)$ and $V_{3 i}(x), i=0,1,2$ and their tilde versions are calculable functions of $x$. Equations (3.23) are the third iterative solutions of (3.11). Proceeding in this way we can obtain the solutions after $n$ successive approximations, which can be written as

$$
\begin{aligned}
& \Delta \Sigma_{n}(x, t)=\sum_{m=0}^{n-1}\left[U_{n m}(x) \frac{\left(\tau-\tau_{0}\right)^{m}}{m !} e^{N_{1}\left(\tau-\tau_{0}\right)}-\tilde{U}_{n m}(x) \frac{\left(\tau-\tau_{0}\right)^{m}}{m !} e^{N_{2}\left(\tau-\tau_{0}\right)}\right] \\
& \Delta G_{n}(x, t)=\sum_{m=0}^{n-1}\left[V_{n m}(x) \frac{\left(\tau-\tau_{0}\right)^{m}}{m !} e^{N_{1}\left(\tau-\tau_{0}\right)}-\tilde{V}_{n m}(x) \frac{\left(\tau-\tau_{0}\right)^{m}}{m !} e^{N_{2}\left(\tau-\tau_{0}\right)}\right]
\end{aligned}
$$

where $U_{n m}(x), \tilde{U}_{n m}(x), V_{n m}(x)$, and $\tilde{V}_{n m}(x)$ are calculable functions of $x$. Equations (3.24) and (3.25) are our main results.

\subsection{Polarized Structure Functions $g_{1}^{p}\left(x, Q^{2}\right), g_{1}^{n}\left(x, Q^{2}\right)$}

We have from (3.1) and (3.3)

$$
\frac{\partial \Delta \Sigma_{q i}(x, t)}{\partial t}=\frac{\alpha_{S}(t)}{2 \pi} \int_{x}^{1} \frac{d z}{z} \Delta P_{q q}\left(\frac{x}{z}\right) \Delta \Sigma_{q i}(x, t)
$$


where

$$
\Delta \Sigma_{q i}(x, t)=\Delta q_{i}(x, t)-\frac{1}{2 n_{f}} \Delta \Sigma(x, t)
$$

We use the small $x$ approximation of splitting function $\Delta P_{i j}(x)$ and the assumption $T(t)^{2}=$ $T_{0} T(t)$ [17] (3.10). With these, (3.26) becomes

$$
\begin{aligned}
\frac{\partial \Delta \Sigma_{q i}(x, t)}{\partial t}= & a_{1} T(t) \Delta \Sigma_{q i}(x, t) \\
& +T(t)\left(h_{1}(x) \int_{x}^{1} \frac{\Delta \Sigma_{q i}(z, t)}{z} d z+h_{2}(x) \int_{x}^{1} \ln z \frac{\Delta \Sigma_{q i}(z, t)}{z} d z\right. \\
& \left.+h_{3}(x) \int_{x}^{1} \ln ^{2} z \frac{\Delta \Sigma_{q i}(z, t)}{z} d z\right)
\end{aligned}
$$

where $a_{1}$ and $h_{i}(x), i=1,2,3$ are known functions of $x$.

Now to obtain the first approximate solution of (3.28) we replace $\Delta \Sigma_{q i}(x, t)$ under the integrals appearing in the right-hand side of (3.28) by its boundary value at $x=1$ [11-13]:

$$
\left.\Delta \Sigma_{q i}(x, t)\right|_{x=1}=0
$$

With this substitution, (3.28) becomes

$$
\frac{\partial \Delta \Sigma_{q i}(x, t)}{\partial t}=a_{1} T(t) \Delta \Sigma_{q i}(x, t)
$$

The solution of (3.30) can be given as

$$
\Delta \Sigma_{q i}(x, t)=C_{q i}(x) e^{N_{3} \tau(t)}
$$

where, $C_{q i}(x)$ is the $x$ dependent constant of integration, $N_{3}=a_{1}$.

Now applying the input distribution at $t=t_{0}$, that is,

$$
\begin{aligned}
\left.\Delta \Sigma_{q i}(x, t)\right|_{t=t_{0}} & =\Delta \Sigma_{q i}\left(x, t_{0}\right) \\
& =\Delta q_{i}\left(x, t_{0}\right)-\frac{1}{2 n_{f}} \Delta \Sigma\left(x, t_{0}\right),
\end{aligned}
$$

the solution (3.31) can be written as

$$
\Delta \Sigma_{1 q i}(x, t)=\Delta \Sigma_{q i}\left(x, t_{0}\right) e^{N_{3}\left(\tau(t)-\tau\left(t_{0}\right)\right)},
$$

where the subscript 1 refers to the first approximate solution. Equation (3.33) is the first approximate solution of (3.28). 
Now using expression (3.33) for $\Delta \Sigma_{1 q i}(x, t)$ in the place of $\Delta \Sigma_{q i}(x, t)$ appearing under the integrals in the right-hand side of (3.28), we have

$$
\frac{\partial \Delta \Sigma_{q i}(x, t)}{\partial t}=a_{1} T(t) \Delta \Sigma_{q i}(x, t)+H_{q 1}(x) T(t) e^{N_{3}\left(\tau(t)-\tau\left(t_{0}\right)\right)},
$$

where

$$
\begin{aligned}
H_{q 1}(x)= & h_{1}(x) \int_{x}^{1} \frac{\Delta \Sigma_{q i}\left(z, t_{0}\right)}{z} d z \\
& +h_{2}(x) \int_{x}^{1} \ln z \frac{\Delta \Sigma_{q i}\left(z, t_{0}\right)}{z} d z+h_{3}(x) \int_{x}^{1} \ln ^{2} z \frac{\Delta \Sigma_{q i}\left(z, t_{0}\right)}{z} d z .
\end{aligned}
$$

Following the method of variation [10] and using the input boundary condition (3.32), we have the second iterative solution of (3.28) as

$$
\Delta \Sigma_{2 q i}(x, t)=\Delta \Sigma_{q i}\left(x, t_{0}\right) e^{N_{3}\left(\tau-\tau_{0}\right)}+H_{q 1}(x)\left(\tau-\tau_{0}\right) e^{N_{3}\left(\tau-\tau_{0}\right)} .
$$

Equation (3.36) is an improvement over (3.33).

Again substituting $\Delta \Sigma_{2 q i}(x, t)$ from (3.36) for $\Delta \Sigma_{q i}(x, t)$ appearing under the integrals in the right-hand side of (3.28), we have

$$
\begin{aligned}
\frac{\partial \Delta \Sigma_{q i}(x, t)}{\partial t}= & a_{1} T(t) \Delta \Sigma_{q i}(x, t)+H_{q 1}(x) T(t) e^{N_{3}\left(\tau-\tau_{0}\right)} \\
& +H_{q 2}(x) T(t)\left(\tau-\tau_{0}\right) e^{N_{3}\left(\tau-\tau_{0}\right)}
\end{aligned}
$$

Proceeding in a similar manner we can obtain the solution of (3.37) as

$$
\begin{aligned}
\Delta \Sigma_{3 q i}(x, t)= & \Delta \Sigma_{q i}\left(x, t_{0}\right) e^{N_{3}\left(\tau-\tau_{0}\right)}+H_{q 1}(x)\left(\tau-\tau_{0}\right) e^{N_{3}\left(\tau-\tau_{0}\right)} \\
& +H_{q 2}(x) \frac{\left(\tau-\tau_{0}\right)^{2}}{2} e^{N_{3}\left(\tau-\tau_{0}\right)}
\end{aligned}
$$

where

$$
H_{q 2}(x)=h_{1}(x) \int_{x}^{1} \frac{H_{q 1}(z)}{z} d z+h_{2}(x) \int_{x}^{1} \ln z \frac{H_{q 1}(z)}{z} d z+h_{3}(x) \int_{x}^{1} \ln ^{2} z \frac{H_{q 1}(z)}{z} d z .
$$

Equation (3.38) is the solution of (3.28) after third approximation. Similarly, the fourth iterative solution will be

$$
\begin{aligned}
\Delta \Sigma_{4 q i}(x, t)= & \Delta \Sigma_{q i}\left(x, t_{0}\right) e^{N_{3}\left(\tau-\tau_{0}\right)}+H_{q 1}(x)\left(\tau-\tau_{0}\right) e^{N_{3}\left(\tau-\tau_{0}\right)} \\
& +H_{q 2}(x) \frac{\left(\tau-\tau_{0}\right)^{2}}{2} e^{N_{3}\left(\tau-\tau_{0}\right)}+H_{q 3}(x) \frac{\left(\tau-\tau_{0}\right)^{3}}{6} e^{N_{3}\left(\tau-\tau_{0}\right)},
\end{aligned}
$$


where

$$
H_{q 3}(x)=h_{1}(x) \int_{x}^{1} \frac{H_{q 2}(z)}{z} d z+h_{2}(x) \int_{x}^{1} \ln z \frac{H_{q 2}(z)}{z} d z+h_{3}(x) \int_{x}^{1} \ln ^{2} z \frac{H_{q 2}(z)}{z} d z .
$$

Proceeding in this way, we have the solution of (3.28) after $n$ approximation as

$$
\Delta \Sigma_{n q i}(x, t)=\sum_{m=0}^{n-1} H_{q m}(x) \frac{\left(\tau-\tau_{0}\right)^{m}}{m !} e^{N_{3}\left(\tau-\tau_{0}\right)},
$$

where

$$
H_{q m}(x)=h_{1}(x) \int_{x}^{1} \frac{H_{q(m-1)}(z)}{z} d z+h_{2}(x) \int_{x}^{1} \ln z \frac{H_{q(m-1)}(z)}{z} d z+h_{3}(x) \int_{x}^{1} \ln ^{2} z \frac{H_{q(m-1)}(z)}{z} d z .
$$

Now using the expression for $\Delta \Sigma_{m}(x, t)$ (3.24), the analytical expression for individual quark distributions $\Delta q_{n i}(x, t)$ after $n$ approximation can be given as

$$
\begin{aligned}
\Delta q_{n i}(x, t)= & \Delta \Sigma_{n q i}(x, t)+\frac{1}{2 n_{f}} \Delta \Sigma_{n}(x, t) \\
=\sum_{m=0}^{n-1}[ & U_{n m}(x) \frac{\left(\tau-\tau_{0}\right)^{m}}{m !} e^{N_{1}\left(\tau-\tau_{0}\right)}-\tilde{U}_{n m}(x) \frac{\left(\tau-\tau_{0}\right)^{m}}{m !} e^{N_{2}\left(\tau-\tau_{0}\right)} \\
& \left.+H_{q m}(x) \frac{\left(\tau-\tau_{0}\right)^{m}}{m !} e^{N_{3}\left(\tau-\tau_{0}\right)}\right] .
\end{aligned}
$$

We now use (3.44) and (3.25) (for $\Delta G_{n}(x, t)$ ) to obtain the analytical expressions for $g_{1}^{p}(x, t)$ and $g_{1}^{n}(x, t)$ in NLO as

$$
\begin{aligned}
g_{1 n}(x, t)=\frac{1}{2} \sum_{q} e_{q}^{2}\left[\Delta q_{n i}(x, t)+\Delta \bar{q}_{n i}(x, t)\right. \\
\left.+\frac{\alpha_{s}(t)}{2 \pi} \int_{x}^{1} \frac{d z}{z}\left(\Delta C_{q}\left(\frac{x}{z}\right)\left(\Delta q_{n i}(z, t)+\Delta \bar{q}_{n i}(z, t)\right)+\Delta C_{G}\left(\frac{x}{z}\right) \Delta G_{n i}(z, t)\right)\right],
\end{aligned}
$$

where subscript $n$ indicates $n$ approximations and $\Delta C_{q}(x)$ and $\Delta C_{G}(x)$ are called Wilson coefficients [15] given in the small $x$ limit as

$$
\begin{aligned}
& \Delta C_{q}(x)=\frac{2}{3}-\frac{4}{3} \ln x \\
& \Delta C_{G}(x)=\frac{3}{2}+\frac{1}{2} \ln x .
\end{aligned}
$$


Equations (3.44) and (3.45) are our main results.

\subsection{Results and Discussion}

Among the several analyses that have included all or most of the present world data on polarized structure functions [18-23] we have used here LSS'05 NLO (MS) input distributions (set-1) at $Q^{2}=1 \mathrm{GeV}^{2}$ [20]. We have taken $n_{f}=3$ and $T_{0}$ of (3.10) to be 0.03 [17].

Initially, as described in Section 4 , we have worked out up to third approximation and obtained the solutions after third approximation (3.23) of the approximate AltarelliParisi equations at small $x$ region (3.11). However, the $x$-dependent parts of the solutions (3.23), namely, $U_{3 i}(x), \tilde{U}_{3 i}(x), V_{3 i}(x)$, and $\tilde{V}_{3 i}(x)$ are two-fold integrations of certain hypergeometric and logarithmic functions (the $n$th approximate solutions involve $(n-1)$ fold such integrations).

To obtain the analytical forms of the fourth approximate solutions, we parametrize the results of the third iteration in the range $10^{-5}<x<1$ (the range of $x$ where LSS'05 numerical results for parton distributions are available) by the following effective functional forms:

$$
\begin{aligned}
& \Delta \Sigma_{3}(x, t)=L_{\Sigma}(x)\left[1+\left(\tau-\tau_{0}\right)+\frac{\left(\tau-\tau_{0}\right)^{2}}{2}\right]\left(\exp \left[N_{1}\left(\tau-\tau_{0}\right)\right]+\exp \left[N_{2}\left(\tau-\tau_{0}\right)\right]\right), \\
& \Delta G_{3}(x, t)=L_{G}(x)\left[1+\left(\tau-\tau_{0}\right)+\frac{\left(\tau-\tau_{0}\right)^{2}}{2}\right]\left(\exp \left[N_{1}\left(\tau-\tau_{0}\right)\right]+\exp \left[N_{2}\left(\tau-\tau_{0}\right)\right]\right),
\end{aligned}
$$

where

$$
\begin{gathered}
L_{\Sigma}(x)=\alpha_{1} x^{\beta_{1}}(1-x)^{\gamma_{1}}\left(1+\delta_{1} x+\xi_{1} x^{\eta_{1}}\right), \\
L_{G}(x)=\alpha_{2} x^{\beta_{2}}(1-x)^{\gamma_{2}}\left(1+\delta_{2} x+\xi_{2} x^{\eta_{2}}\right), \\
\alpha_{1}=0.396, \quad \beta_{1}=0.693, \quad \gamma_{1}=3.046, \quad \delta_{1}=2.86, \quad \xi_{1}=-12.049, \quad \eta_{1}=4.82, \\
\alpha_{2}=3.522, \quad \beta_{2}=1.88, \quad \gamma_{2}=2.59, \quad \delta_{2}=-2.81, \quad \xi_{2}=1.61, \quad \eta_{2}=0.043 .
\end{gathered}
$$

Using (3.47) we get the following approximate analytic forms after fourth approximation:

$$
\begin{aligned}
\Delta \Sigma_{4}(x, t)=[ & U_{40}(x)+U_{41}(x)\left(\tau-\tau_{0}\right)+U_{42}(x) \frac{\left(\tau-\tau_{0}\right)^{2}}{2} \\
& \left.+U_{43}(x) \frac{\left(\tau-\tau_{0}\right)^{3}}{6}\right] \exp \left[N_{1}\left(\tau-\tau_{0}\right)\right] \\
& -\left[\tilde{U}_{40}(x)+\tilde{U}_{41}(x)\left(\tau-\tau_{0}\right)+\tilde{U}_{42}(x) \frac{\left(\tau-\tau_{0}\right)^{2}}{2}\right. \\
& \left.+\tilde{U}_{43}(x) \frac{\left(\tau-\tau_{0}\right)^{3}}{6}\right] \exp \left[N_{2}\left(\tau-\tau_{0}\right)\right],
\end{aligned}
$$




$$
\begin{aligned}
\Delta G_{4}(x, t)=[ & V_{40}(x)+V_{41}(x)\left(\tau-\tau_{0}\right)+V_{42}(x) \frac{\left(\tau-\tau_{0}\right)^{2}}{2} \\
& \left.+V_{43}(x) \frac{\left(\tau-\tau_{0}\right)^{3}}{6}\right] \exp \left[N_{1}\left(\tau-\tau_{0}\right)\right] \\
& -\left[\tilde{V}_{40}(x)+\tilde{V}_{41}(x)\left(\tau-\tau_{0}\right)+\tilde{V}_{42}(x) \frac{\left(\tau-\tau_{0}\right)^{2}}{2}\right. \\
& \left.+\tilde{V}_{43}(x) \frac{\left(\tau-\tau_{0}\right)^{3}}{6}\right] \exp \left[N_{2}\left(\tau-\tau_{0}\right)\right] .
\end{aligned}
$$

The expressions for the $x$-dependent functions appearing in (3.49) are calculable.

We now compare our solutions after fourth approximation with the LSS'05 numerical results (NLO (MS), set-1) at $Q^{2}=10 \mathrm{GeV}^{2}$ (Figures 1 and 2).

We observe that with more and more iterations our analytic solutions come closer to the LSS'05 numerical results.

However, the values of $\Delta \Sigma\left(Q^{2}\right)$ and $\Delta G\left(Q^{2}\right)$ defined as

$$
\begin{aligned}
& \Delta \Sigma\left(Q^{2}\right)=\int_{0}^{1} \Delta \Sigma\left(x, Q^{2}\right) d x \\
& \Delta G\left(Q^{2}\right)=\int_{0}^{1} \Delta G\left(x, Q^{2}\right) d x
\end{aligned}
$$

obtained from our solutions for $\Delta \Sigma\left(x, Q^{2}\right)$ and $\Delta G\left(x, Q^{2}\right)$ at $Q^{2}=10 \mathrm{GeV}^{2}$ in NLO are found to be higher than the corresponding experimental values [23-28].

There may be two sources from where some errors have crept in.

(1) As the parametrizations were done only in the range $10^{-5}<x<1$ (as in LSS'05), it may not be adequate in calculating the integrated quantities like $\Delta \Sigma\left(Q^{2}\right)$ and $\Delta G\left(Q^{2}\right)$ which involve integrations of our solutions (3.49) in the range $0<x<1$.

(2) We obtained the solutions of AP equations with small $x$ approximation. The disagreement of the integrated quantities with the experimental values, as observed in Table 1, perhaps indicates that incorporation of high $x$ effect is important.

In the case of polarized quark distributions with individual flavour also, we have initially worked up to third iterative solutions (3.38). The fourth iterative solution (3.40) contains terms like $H_{q 3}(x)$ which can be obtained by evaluating the three integrals as given by (3.41). As such integrals involve several hypergeometric functions $H_{q 2}(x)$ along with logarithmic functions, to proceed for approximate fourth iterative analytical solution, we simplify it by performing the parametrizations in the range $10^{-5}<x<1$ (the range of $x$ 
Table 1: Values of $\Delta \sum\left(Q^{2}\right)$ and $\Delta G\left(Q^{2}\right)$ obtained from this work in NLO after four iterations at $Q^{2}=10 \mathrm{GeV}^{2}$.

\begin{tabular}{cccccc}
\hline & 1st iter. & 2nd iter. & 3rd iter. & 4th iter. & Exp. values \\
\hline$\Delta \Sigma\left(Q^{2}\right)$ & 0.348 & 0.427 & 0.646 & 0.631 & $\begin{array}{c}0.35 \pm 0.06 \\
\left(\text { COMPASS at } Q^{2}=3 \mathrm{GeV}^{2}\right) \\
0.33 \pm 0.04 \\
\left(\text { HERMES at } Q^{2}=5 \mathrm{GeV}^{2}\right)\end{array}$ \\
\hline$\Delta G\left(Q^{2}\right)$ & 0.134 & 0.342 & 0.458 & 0.535 & $\begin{array}{c}0.29 \pm 0.32 \\
\text { (From all fits of present data) }\end{array}$ \\
\hline
\end{tabular}

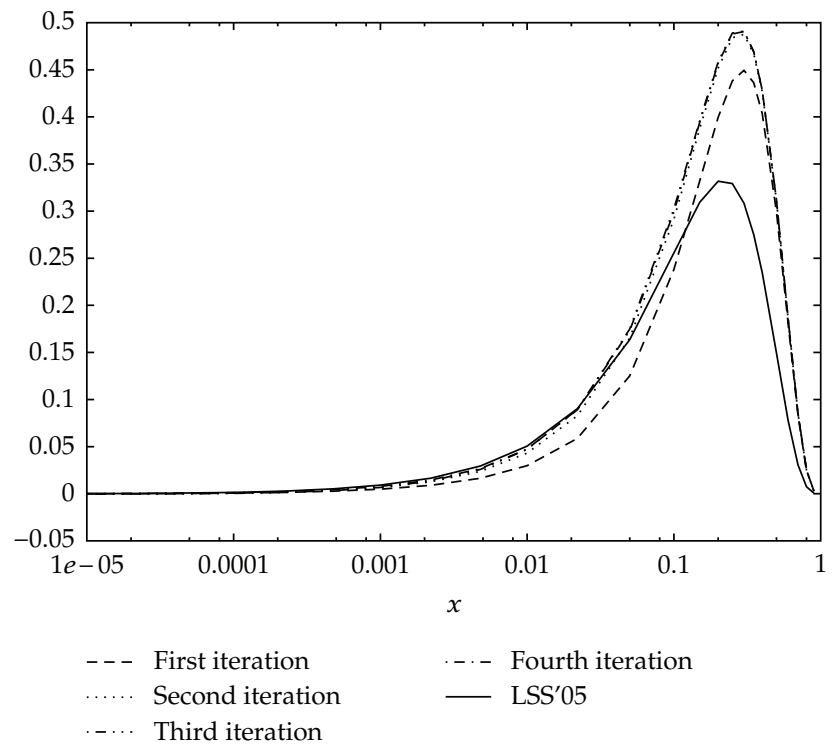

Figure 3: $x \Delta u(x)$ in $\mathrm{NLO}$ at $Q^{2}=10 \mathrm{GeV}^{2}$.

where LSS'05 numerical results for parton distributions are available) to get the following effective expressions for $H_{q 2}(x)$

$$
\begin{aligned}
& H_{u 2}(x)(\text { for } u \text { quark })=103.04 x^{-0.15}(1-x)^{8.33}\left(1+0.477 x-1.158 x^{0.096}\right), \\
& H_{d 2}(x)(\text { for } d \text { quark })=-57.27 x^{-0.19}(1-x)^{9.47}\left(1+0.524 x-1.166 x^{0.088}\right), \\
& H_{s 2}(x)(\text { for } s \text { quark })=-15.73 x^{-0.064}(1-x)^{7.63}\left(1+0.393 x-1.143 x^{0.095}\right)
\end{aligned}
$$

We have obtained the fourth iterative solutions by using these effective expressions for $H_{q 2}(x)$, where $q=u, d, s$ as shown by (3.51). We now compare our work for polarized flavour specific quark distributions with the LSS'05 NLO (MS), set-1 numerical results at $Q^{2}=10 \mathrm{GeV}^{2}$ (Figures 3, 4, and 5). We have observed that increasing iterations bring our solutions for individual quark densities closer to the numerical results at small $x$. In the high $x$ range our results however deviate from the numerical results probably due to the use of 


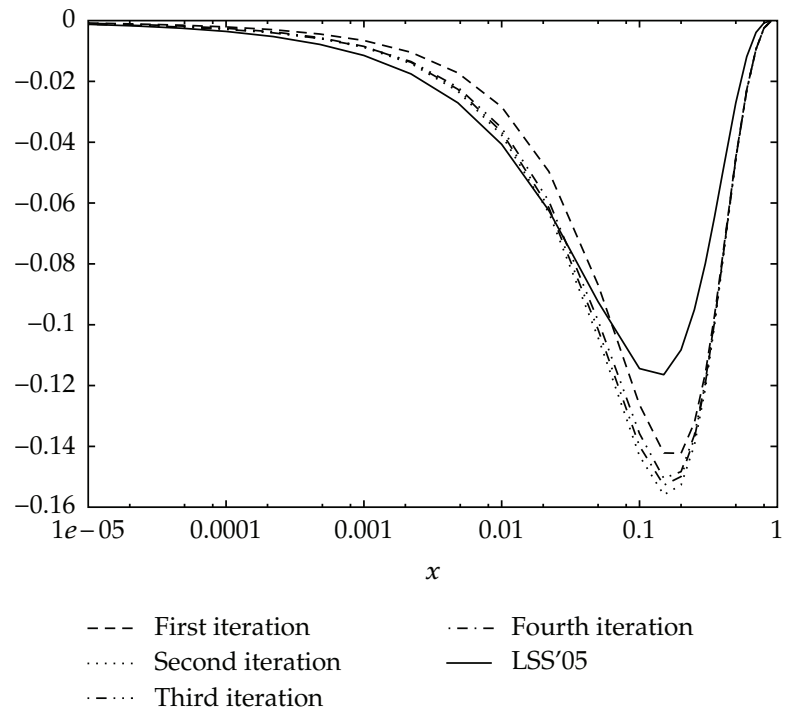

Figure 4: $x \Delta d(x)$ in NLO at $Q^{2}=10 \mathrm{GeV}^{2}$.

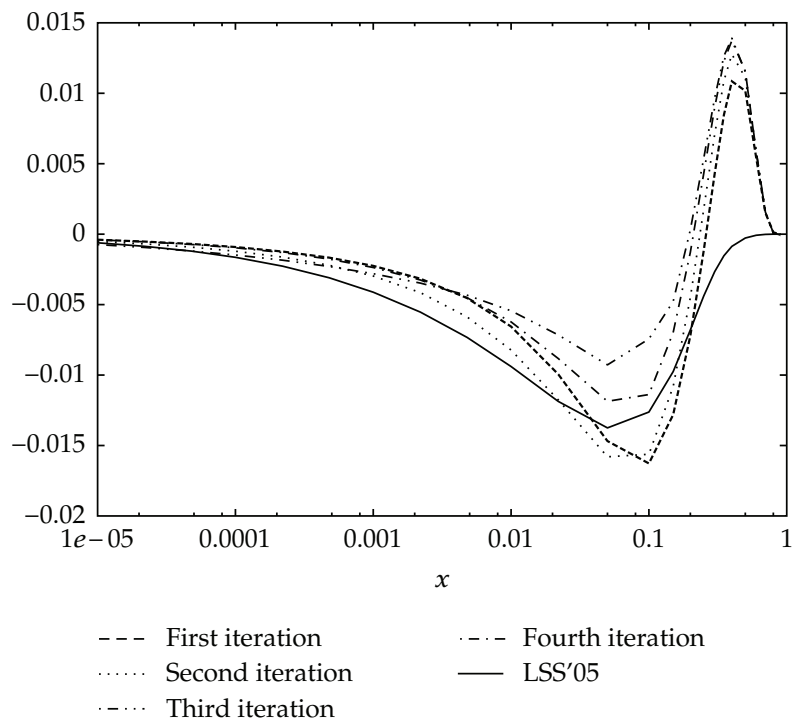

Figure 5: $x \Delta s(x)$ in NLO at $Q^{2}=10 \mathrm{GeV}^{2}$.

small $x$ splitting functions. Another observation is that while the numerical (LSS'05) result gives negative $\Delta s(x)$, our solution for $\Delta s(x)$ becomes positive beyond $x \approx 0.3$.

We also record the values of the strange quark contribution $\Delta S=\int_{0}^{1}(\Delta s(x)+\Delta \bar{s}(x)) d x$ (Table 2) towards the spin of the proton at $Q^{2}=10 \mathrm{GeV}^{2}$ in different iterations to be compared with the experimental values [21,22].

We now use our formalism in the next to leading order (NLO) to calculate the structure functions $g_{1}^{p}(x, t)$ and $g_{1}^{n}(x, t)$ as given by (3.45) and compare them with the LSS'05 numerical 


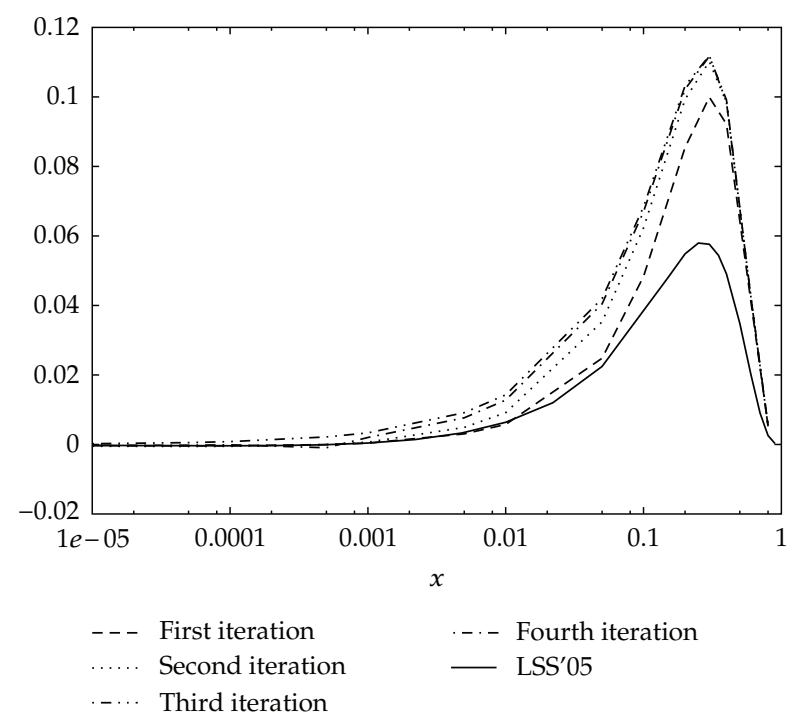

Figure 6: Plot for $x g_{1}^{p}\left(x, Q^{2}\right)$ in NLO at $Q^{2}=10 \mathrm{GeV}^{2}$.

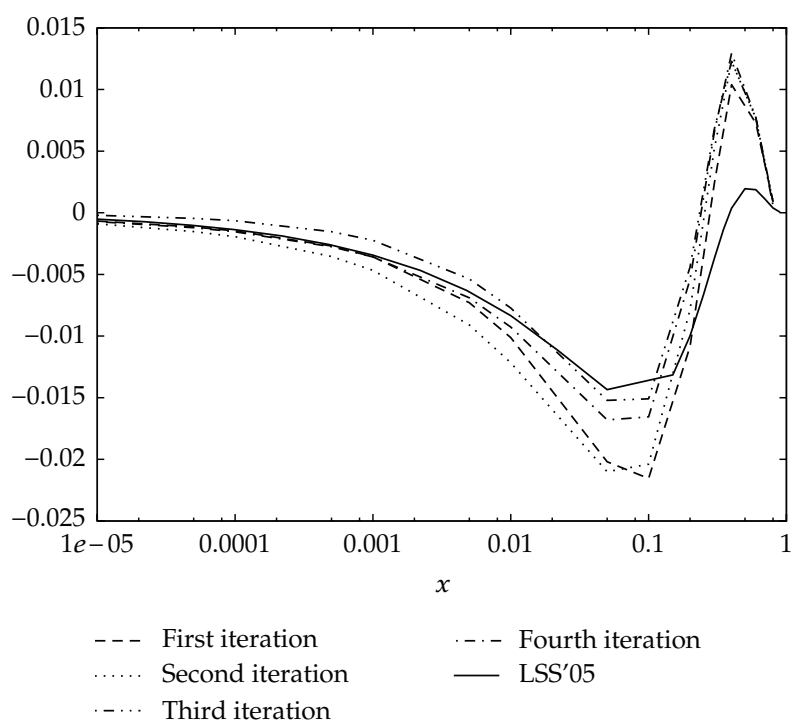

Figure 7: Plot for $x g_{1}^{n}\left(x, Q^{2}\right)$ in NLO at $Q^{2}=10 \mathrm{GeV}^{2}$.

results (Figures 6 and 7). It is observed that our approximate analytical results are compatible with that obtained numerically (LSS'05).

\section{Conclusion}

QCD analysis of the quark and gluon contributions towards the spin of the nucleon in the small $x$ region is very important for a clear understanding of the spin structure of the nucleon and this is mainly done through the Altarelli-Parisi [1-3] evolution equations. In this work 
Table 2: Values of $\Delta S$ obtained from this work in NLO after four iterations at $Q^{2}=10 \mathrm{GeV}^{2}$.

\begin{tabular}{|c|c|c|c|c|c|c|}
\hline & 1st iter. & 2nd iter. & 3rd iter. & 4th iter. & $\mathrm{LSS}^{\prime} 06(\overline{\mathrm{MS}})$ & DSSV $(\overline{\mathrm{MS}})$ \\
\hline$\Delta S$ & -0.045 & -0.049 & -0.0145 & -0.033 & $-0.126 \pm 0.010$ & -0.114 \\
\hline
\end{tabular}

we have given a formalism based on the method of successive approximations, for obtaining analytical solutions in the next to leading order (NLO), valid in small $x$ region.

In Section 2 we have given a method for solving a system of two first order linear homogeneous differential equations with variable coefficients.

In Section 3 we have obtained approximate analytical solutions of Altarelli-Parisi equations for the polarized singlet quark density $\Delta \Sigma(x, t)$ and polarized gluon density $\Delta G(x, t)$ in the small $x$ limit at NLO by using a method described in Section 2 along with the method of iteration. It is observed that, with increasing number of iterations, the solutions approach the numerical results, specifically in the small $x$ region. We have also given the analytical expressions for the individual polarized quark densities $\Delta q(x, t)$ and using them we have obtained the expressions for the polarized structure functions $g_{1}^{p}(x, t)$ and $g_{1}^{n}(x, t)$. Our results are found to be compatible with those obtained numerically (LSS'05). It is possible that such agreement will improve if the assumption $T(t)^{2}=T_{0} T(t)$ (3.10) can be removed and it will be our attempt in the future communication.

\section{Appendix}

Consider

$$
\begin{gathered}
\Delta P_{q q 0}\left(n_{f}\right)=\left(\frac{\pi^{2}}{3}-9\right) C_{F}^{2}+\left(\frac{223}{18}-\frac{\pi^{2}}{3}\right) C_{F} C_{A}-\frac{4}{9} C_{F} T_{f} \\
\Delta P_{q q 1}\left(n_{f}\right)=-5 C_{F}^{2}+\frac{23}{6} C_{F} C_{A}-\frac{8}{3} C_{F} T_{f} \\
\Delta P_{q q 2}\left(n_{f}\right)=-\frac{3 C_{F}^{2}}{2}+C_{F} C_{A}-2 C_{F} T_{f} \\
\Delta P_{q g 0}\left(n_{f}\right)=\left(\frac{2 \pi^{2}}{3}-22\right) C_{F} T_{f}+\left(24-\frac{2 \pi^{2}}{3}\right) C_{A} T_{f} \\
\Delta P_{q g 1}\left(n_{f}\right)=2 C_{A} T_{f}-9 C_{F} T_{f} \\
\Delta P_{q g 2}\left(n_{f}\right)=-C_{F} T_{f}-2 C_{A} T_{f} \\
\Delta P_{g q 0}\left(n_{f}\right)=-\frac{17}{2} C_{F}^{2}+\frac{41}{9} C_{F} C_{A}-\frac{16}{9} C_{F} T_{f} \\
\Delta P_{g q 1}\left(n_{f}\right)=-2 C_{F}^{2}+4 C_{F} C_{A} \\
\Delta P_{g q 2}\left(n_{f}\right)=C_{F}^{2}+2 C_{F} C_{A} \\
\Delta P_{g g 0}\left(n_{f}\right)=\frac{97}{18} C_{A}^{2}-\frac{76}{9} C_{A} T_{f}-10 C_{F} T_{f}
\end{gathered}
$$




$$
\begin{gathered}
\Delta P_{g g 1}\left(n_{f}\right)=\frac{29}{3} C_{A}^{2}-\frac{4}{3} C_{A} T_{f}-10 C_{F} T_{f}, \\
\Delta P_{g g 2}\left(n_{f}\right)=4 C_{A}^{2}-2 C_{F} T_{f},
\end{gathered}
$$

where $C_{F}=4 / 3, C_{A}=3$ and $T_{f}=n_{f} T_{R}=n_{f} / 2$.

Therefore,

$$
\begin{aligned}
H_{3}(x)= & h_{1}(x) \int_{x}^{1} \frac{L_{\Sigma}(z)}{z} d z+h_{2}(x) \int_{x}^{1} \frac{L_{\Sigma}(z)}{z} \ln z d z+h_{3}(x) \int_{x}^{1} \frac{L_{\Sigma}(z)}{z} \ln ^{2} z d z \\
& +k_{1}(x) \int_{x}^{1} \frac{L_{G}(z)}{z} d z+k_{2}(x) \int_{x}^{1} \frac{L_{G}(z)}{z} \ln z d z+k_{3}(x) \int_{x}^{1} \frac{L_{G}(z)}{z} \ln ^{2} z d z, \\
K_{3}(x)= & p_{1}(x) \int_{x}^{1} \frac{L_{\Sigma}(z)}{z} d z+p_{2}(x) \int_{x}^{1} \frac{L_{\Sigma}(z)}{z} \ln z d z+p_{3}(x) \int_{x}^{1} \frac{L_{\Sigma}(z)}{z} \ln ^{2} z d z \\
& +q_{1}(x) \int_{x}^{1} \frac{L_{G}(z)}{z} d z+q_{2}(x) \int_{x}^{1} \frac{L_{G}(z)}{z} \ln z d z+q_{3}(x) \int_{x}^{1} \frac{L_{G}(z)}{z} \ln ^{2} z d z .
\end{aligned}
$$

\section{References}

[1] G. Altarelli and G. Parisi, “Asymptotic freedom in parton language,” Nuclear Physics B, vol. 126, no. 2, pp. 298-318, 1977.

[2] Yu. L. Dokshitzer, "Calculation of the structure functions for deep inelastic scattering and $\mathrm{e}^{+} \mathrm{e}^{-}$annihilation by perturbation theory in quantum chromodynamics," Soviet Physics_JETP, vol. 46, p. 641, 1977.

[3] V. N. Gribov and L. N. Lipatov, “Deep inelastic e p scattering in perturbation theory,” Soviet Journal of Nuclear Physics, vol. 15, p. 438, 1972.

[4] EIC Collaboration, September 2010, http://web.mit.edu/eicc/Documentation.html.

[5] N. A. Khorramian, S. A. Tehrani, S. T. Monfared, F. Arbabifar, and F. I. Olness, "Polarized deeply inelastic scattering (DIS) structure functions for nucleons and nuclei," Physical Review D, vol. 83, no. 5, Article ID 054017, 11 pages, 2011.

[6] E. Leader, A. V. Sidorov, and D. B. Stamenov, "Polarized parton densities in the nucleon," Physical Review D, vol. 58, no. 11, Article ID 114028, 1998.

[7] A. V. Sidorov, E. Leader, and D. B. Stamenov, "NLO QCD analysis of polarized deep inelastic scattering," International Journal of Modern Physics A, vol. 13, pp. 5573-5592, 1998.

[8] V. G. Krivokhizhin, "QCD analysis of structure functions in terms of Jacobi polynomials," Zeitschrift für Physik C, vol. 36, no. 1, pp. 51-59, 1987.

[9] S. T. Monfared, A. N. Khorramian, F. Arbabifar, and S. A. Tehrani, "The spin dependent parton distribution functions and their moments," Acta Physica Polonica B, vol. 41, no. 12, pp. 2921-2927, 2010.

[10] G. F. Simmons, Differential Equations, Tata McGraw-Hill, New Delhi, India, 1979.

[11] R. G. Roberts, The Structure of Proton, Cambridge University Press, Cambridge, UK, 1990.

[12] Philip G. Ratcliffe, "Matrix approach to a numerical solution of the Dokshitzer-Gribov-LipatovAltarelli-Parisi evolution equations," Physical Review D, vol. 63, no. 11, Article ID 116004, 7 pages, 2001.

[13] F. J. Yndurain, The Theory of Quark and Gluon Interactions, Springer, Berlin, Germany, 1992.

[14] G. Ridolfi, R. D. Ball, and S. Forte, "Scale dependence and small- $\chi$ behaviour of polarized parton distributions," Nuclear Physics B, vol. 444, no. 1-2, pp. 287-309, 1995.

[15] B. Lampe and E. Reya, "Spin physics and polarized structure functions," MPI-PhT/98-23 DO-TH 98/02, 1998. 
[16] T. Gehrmann and W. J. Stirling, "Analytic approaches to the evolution of polarised parton distributions at small x," Physics Letters B, vol. 365, no. 1-4, pp. 347-358, 1996.

[17] A. Deshamukhya and D. K. Choudhury, "Non-singlet structure function beyond leading order," in Proceedings of the 2nd Regional Conference of Physics Academy of North East, 2000.

[18] M. Hirai, S. Kumano, and N. Saito, "Determination of polarized parton distribution functions and their uncertainties," Physical Review D, vol. 69, no. 5, Article ID 054021, 10 pages, 2004.

[19] J. Blumlein and H. Bottcher, "QCD analysis of polarized deep-inelastic scattering data and parton distributions," Nuclear Physics B, vol. 636, no. 1-2, pp. 225-263, 2002.

[20] A. V. Sidorov, E. Leader, and D. B. Stamenov, "Role of positivity constraints in determining polarized parton densities," Journal of High Energy Physics, vol. 6, 2005.

[21] E. Leader, A. V. Sidorov, and D. B. Stamenov, "Impact of CLAS and COMPASS data on polarized parton densities and higher twist," Physical Review D, vol. 75, no. 7, Article ID 074027, 10 pages, 2007.

[22] M. Stratmann, D. de Florian, R. Sassot, and W. Vogelsang, "Global analysis of helicity parton densities and their uncertainties," Physical Review Letters, vol. 101, no. 7, Article ID 072001, 4 pages, 2008.

[23] V. Y. Alexakhin, Yu. Alexandrov, G. D. Alexeev et al., "Spin asymmetry $A_{1}^{d}$ and the spin-dependent structure function $g_{1}^{d}$ of the deuteron at low values of $x$ and $Q^{2}$, , Physics Letters B, vol. 647, no. 5-6, pp. 330-340, 2007.

[24] A. Airapetian and The HERMES Collaboration, "Single-spin asymmetries in semi-inclusive deep-inelastic scattering on a transversely polarized hydrogen target," Physical Review Letters, vol. 94, no. 1, Article ID 012002, 6 pages, 2005.

[25] A. Airapetian and The HERMES Collaboration, "Measurement of parton distributions of strange quarks in the nucleon from charged-kaon production in deep-inelastic scattering on the deuteron," Physics Letters B, vol. 666, no. 5, pp. 446-450, 2008.

[26] A. Airapetian and The HERMES Collaboration, "Precise determination of the spin structure function $\mathrm{g}_{1}$ of the proton, deuteron, and neutron," Physical Review D, vol. 75, no. 1, Article ID 012007, 48 pages, 2007.

[27] A. Airapetian and The HERMES Collaboration, "Quark helicity distributions in the nucleon for up, down, and strange quarks from semi-inclusive deep-inelastic scattering," Physical Review D, vol. 71, no. 1, Article ID 012003, 36 pages, 2005.

[28] M. Alekseev, V. Yu. Alexakhin, Yu. Alexandrov et al., “The polarised valence quark distribution from semi-inclusive DIS," Physics Letters B, vol. 660, no. 5-6, pp. 458-465, 2008. 

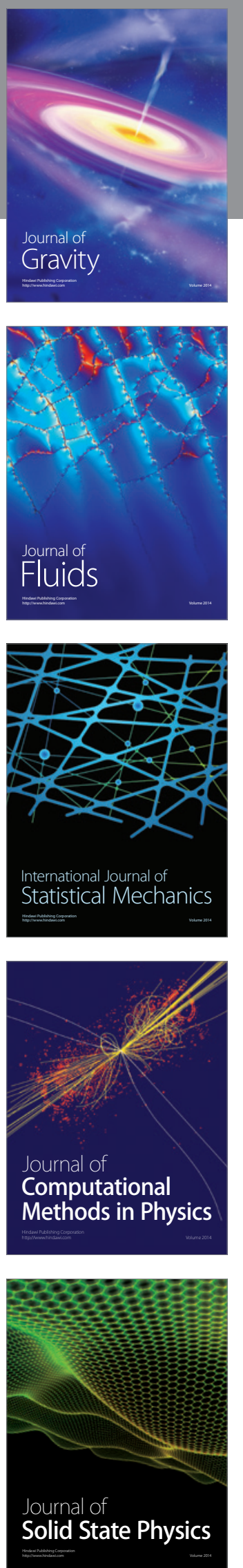
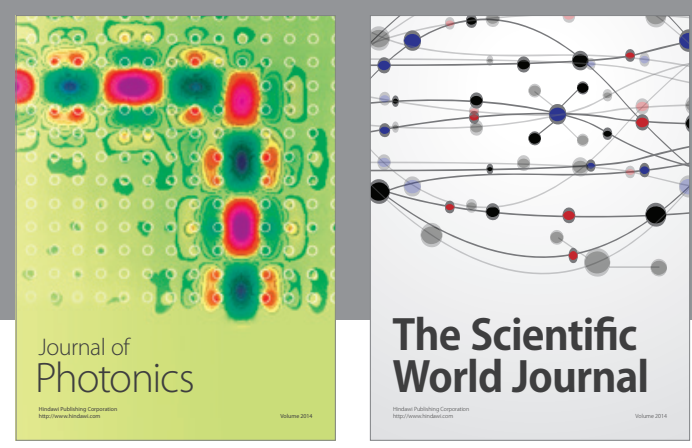

The Scientific World Journal

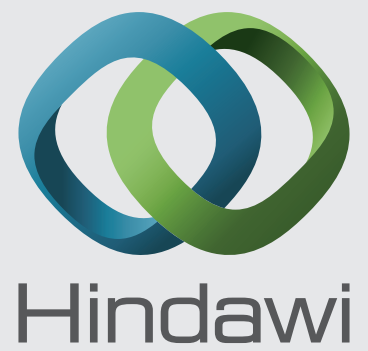

Submit your manuscripts at http://www.hindawi.com
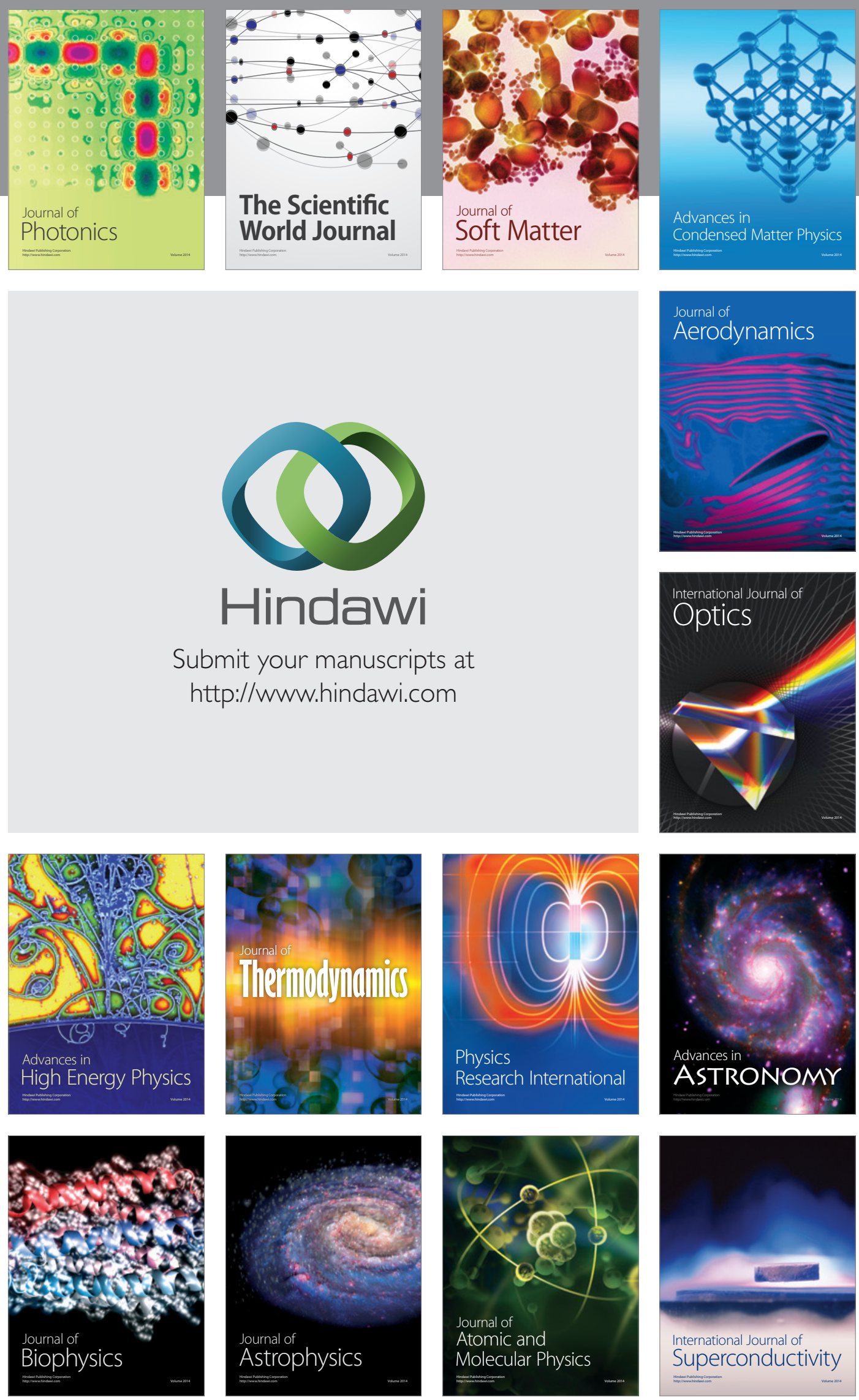
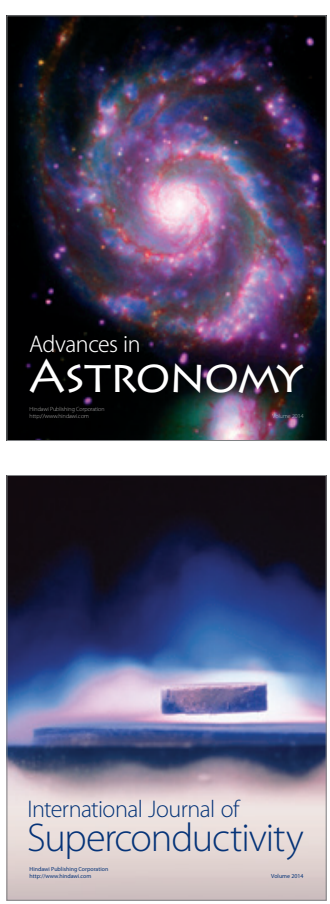